\title{
A NEW SPECIES OF PHLEBIAPORUS (HYMENOPTERA: SCELIONIDAE: TELENOMINAE) FROM INDIA, WITH FIRST DESCRIPTION OF THE MALE OF THE GENUS
}

\author{
Veenakumari Kamalanathan* and Prashanth Mohanraj \\ National Bureau of Agricultural Insect Resources \\ P.B. No. 2491, Hebbal, Bengaluru, 560024 India; *E-mail: veenapmraj@gmail.com
}

A new species of Phlebiaporus Kozlov is described and illustrated. The previously unknown male of the genus is also described. This genus is reported for the first time from the Oriental region and redescribed with additional characters.

Key words: Platygastroidea, Oriental, Palaearctic, Phlebiaporus dichrous.

\section{INTRODUCTION}

Phlebiaporus Kozlov, a monotypic genus, was described with type species P. dichrous Kozlov, 1967 based on specimens collected from the Palaearctic region (Uzbekistan and Kazakhstan). Kozlov (1967) was of the opinion that Phlebiaporus was most similar to the genus Protelenomus Kieffer because of the presence of the following three character states - absence of setae on eyes, absence of postmarginalis and female antennae with 11 antennomeres. The third character, however, is common to all Telenominae. Phlebiaporus was distinguished from Protelenomus Kieffer using the following character states. In Phlebiaporus, the female antenna is clubbed; the stigmalis is very short with small irregular protuberances and is almost perpendicular to the marginalis, marginalis $2 \times$ as long as stigmalis; the metatarsus is not elongate, tarsomeres 2-4 are longer than wide; metasoma is cylindrical not dorsoventrally compressed. In Protelenomus, the female antenna is not clubbed; the stigmalis is long, not perpendicular to marginalis but slanted, marginalis shorter than stigmalis; metatarsus $2 \times$ as long as the combined length of the other tarsi, tarsomeres 2-4 are wider than long; metasoma is dorso-ventrally compressed (Kozlov 1967, Kozlov \& KoNonova 1983). However recent studies on Protelenomus conducted by the senior author reveal that the postmarginalis is very long and distinct in some species and the apical antennomeres in female are incrassate. MAsner (1980), in his key to the Holarctic genera of Scelionidae placed Phlebiaporus closer to Eumicrosoma Gahan because of the presence of a distinctly longer marginalis (2-4× as long as the stigmalis), lack of the postmarginalis, and the usually xanthic metasoma. He distinguished Eumicrosoma from Phlebiaporus using the following character states. In Eumicrosoma the body is dorso-ventrally flattened; the fore wing is narrow and strip-like, marginal cilia are longer or subequal to fore wing width; 
the marginalis is $3-4 \times$ as long as the stigmalis, which is short and slanted. In Phlebiaporus, on the other hand, the body is cylindrical; the fore wing is not narrow, marginal cilia are not longer than $1 / 3$ width of the fore wing; the marginalis at most $2 \times$ as long as the stigmalis, the stigmalis is perpendicular to marginalis. The genus is redescribed and a new species is described from the Oriental region. The male of the genus is described for the first time.

\section{MATERIAL AND METHODS}

Morphological terminology follows Masner (1980) and Miкó et al. (2007, 2010, 2016). All the specimens were collected by yellow pan traps (YPT), pit fall traps (PFT) and sweep netting (SN). These specimens were mounted on point-card tips. The descriptions, measurements and imaging were carried out with a Leica M205A automontage stereomicroscope, with $1 \times$ objective and Leica DFC-500 digital camera. The images were stacked using Leica Application Suite (LAS) software. The holotype and all the paratypes are deposited at the National Bureau of Agricultural Insect Resources, Bengaluru.

The following abbreviations are used in the description of the taxa. All the measurements are taken as per Miкó et al. (2010). HL = head length; $\mathrm{HW}=$ head width; $\mathrm{HH}=$ head height; FCI (frontal cephalic index) $=\mathrm{HW} / \mathrm{HH}$; LCI (lateral cephalic index) $=\mathrm{HH}$ / $\mathrm{HL} ; \mathrm{A} 1-\mathrm{A} 12$ = antennomeres $1-12(\mathrm{~A} 1$ = scape, $\mathrm{A} 2$ = pedicel $) ; \mathrm{L}=$ length; $\mathrm{W}$ = width; $\mathrm{H}=$ height; $\mathrm{OOL}=$ ocular ocellar line; $\mathrm{POL}=$ posterior ocellar line; $\mathrm{LOL}=$ lateral ocellar line; IOS = interorbital space; T1-T2 = Metasomal tergites 1 to 2 . Widths of all metasomal tergites taken anteriorly.

\section{TAXONOMY}

\section{Redescription of the genus Phlebiaporus Kozlov, 1967}

Phlebiaporus Kozlov, 1967: 719 (original description. Type: Phlebiaporus dichrous Kozlov, by monotypy. Diagnosis).

Eyes without setae; head finely reticulate (sometimes frons smooth); female antenna with 11 antennomeres; lateral ocelli either contiguous or close to eyes; mandible bidentate; clypeus triangular; malar sulcus joins the base of eye forming a distinct angle with the orbital furrow; mesoscutum finely reticulate, mesoscutellum smooth; notauli absent; mesepimeral sulcus absent; prespecular sulcus either absent or very weakly foveate; fore wing with submarginalis, marginalis and stigmalis; postmarginalis absent; marginalis at least $2 \times$ as long as stigmalis; both marginalis and stigmalis with small irregular protuberances; stigmalis either perpendicular or slanted to marginalis; fore wing and marginal cilia at most 1/3 width of wing; hind wing marginal cilia either subequal or longer than wing width; metasoma partially or entirely xanthic, cylindrical not dorso-ventrally compressed. Male similar to female with $1 / 3$ of posterior margin of $\mathrm{T} 2$ and remaining tergites brownishblack; antenna with 12 elongate antennomeres.

Diagnosis - Combination of character states, viz., body cylindrical, marginalis at least $2 \times$ as long as stigmalis, marginalis and stigmalis with small 
irregular protuberances, fore wing and hind wing marginal cilia extremely long, distinguishes this genus from all other telenomine genera.

\section{KEY TO PHLEBIAPORUS KIEFFER AND ITS RELATED GENERA}

1. Stigmalis longer than marginalis; metatarsus $2 \times$ as long as the combined lengths of other tarsi, tarsomeres 2-4, wider than long; metasoma dorsoventrally compressed; postmarginalis either present or absent; phoretic species

Protelenomus Kieffer, 1906

- $\quad$ Marginalis at least $2 \times$ as long as stigmalis; metatarsus not unusually elongate, tarsomeres 2-4, longer than wide; metasoma not dorsoventrally compressed, but cylindrical; post marginalis absent; phoresy not known 2

2. Body dorso-ventrally flattened at least $2 \times$ as wide as high; fore wing narrow and strip-like, marginal cilia longer or subequal to wing width; marginalis $3-4 \times$ as long as stigmalis

Eumicrosoma Gahan, 1913

- Body cylindrical, as high as wide; fore wing not narrow, marginal cilia not longer than $1 / 3$ width of wing; marginalis at most $2.3 \times$ as long as stigmalis

Phlebiaporus Kozlov, 1967

Phlebiaporus supattra Veenakumari sp. n.

(Figs 1-14)

Diagnosis - This species differs from $P$. dichrous (Fig. 14) in the following character states. In $P$. dichrous stigmalis almost perpendicular to marginalis; marginalis at most $2 \times$ as long as stigmalis; fore wing without medial black band; submarginalis at least $4 \times$ as long as marginalis; head and mesosoma dark brown, metasoma yellow-brown; prespecular sulcus weakly foveate; diameter of the scutes on head and mesoscutum subequal; scutoscutellar sulcus narrow. In $P$. supattra sp. n. stigmalis not perpendicular but at an acute angle to marginalis; marginalis at least $2.2 \times$ as long as stigmalis; fore wing with a distinct medial broad black band (0.92× width of wing); submarginalis at most $2.8 \times$ as long as marginalis; entire habitus orange-brown; prespecular sulcus not foveate; diameter of scutes on head smaller than those on mesoscutum; scutoscutellar sulcus very wide.

Description: Holotype: Female: Body length $=0.94 \mathrm{~mm}$; $(\mathrm{m}=0.95(0.89-1.02) \mathrm{mm}$, $\mathrm{SD}=0.04, \mathrm{n}=20$ )

Colour (Fig. 1). Head and mesosoma orangish brown, metasoma orangish yellow, T1 darker than T2; posterior tergites black; eyes black, ocelli silvery grey with black patch towards inner side; radicle, A1-A2 golden yellow, remaining antennomeres brownish black 
except paler A6-A7; mandible yellow with teeth reddish brown; interantennal process orange; legs yellow.

Head (Figs 3, 4, 8, 9). FCI = 1.40; LCI = 1.21; IOS 0.50× width of head, 1.15× length of eye; frons, vertex and gena finely reticulate; scutes smaller than those on mesoscutum; reticulations on occiput distinct; POL and LOL in ratio of 12.3:6.7; lateral ocelli contiguous
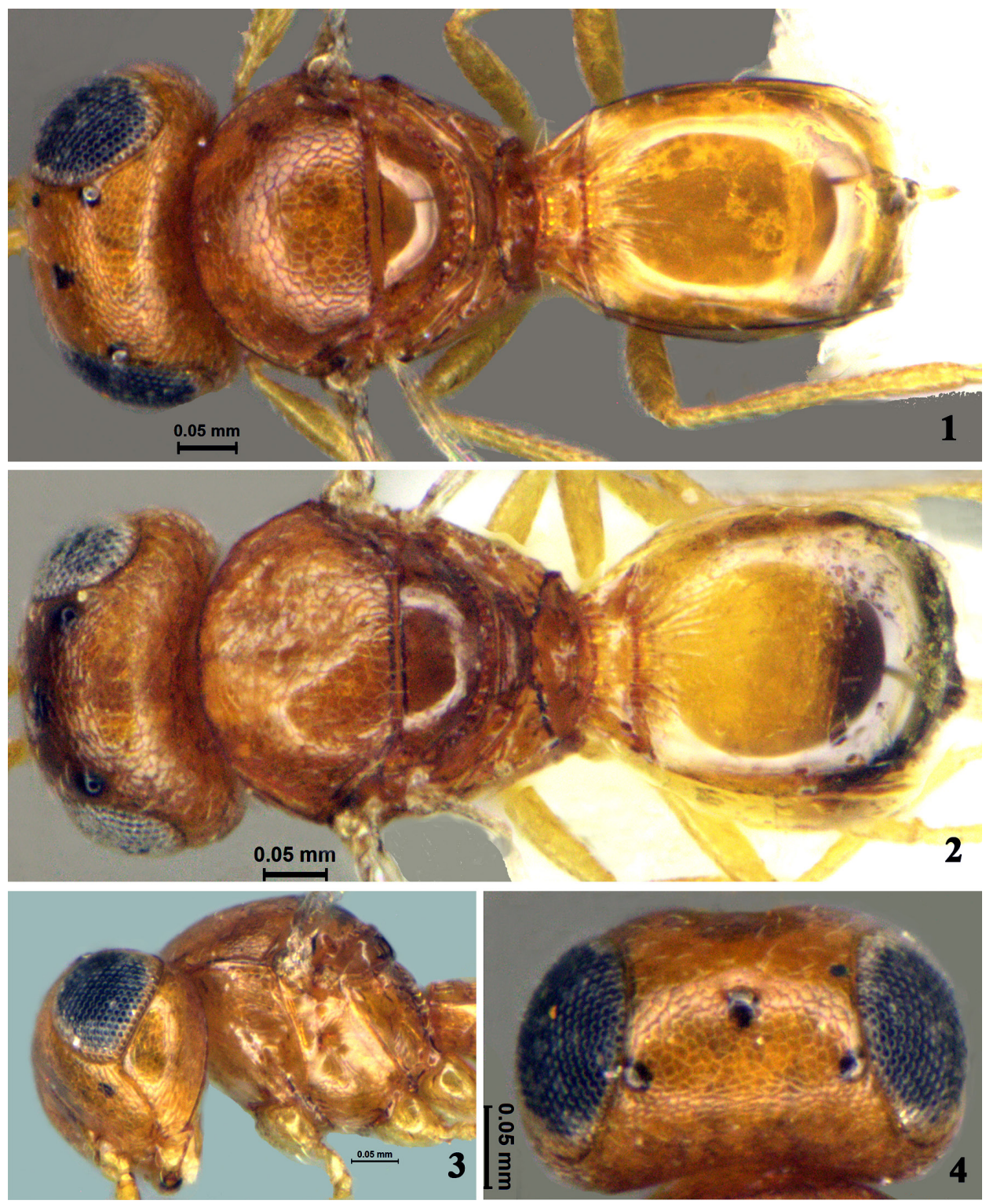

Figs 1-4. Bright field images of Phlebiaporus supattra sp. n.: $1=$ dorsal view of habitus (female), 2 = dorsal view of habitus (male), 3 = head and pleuron, $4=$ head showing ocelli 
with eye margin; eyes large ( $\mathrm{L}: \mathrm{W}=12.6: 11.8)$; central keel present; mandible (L: $\mathrm{W}=2.3: 5.9)$ with both teeth identical; clypeus triangular ( $\mathrm{L}: \mathrm{W}=3.5: 6.1)$; interantennal process short and beak-like; radicle $0.24 \times$ the length of $\mathrm{A} 1$.

Mesosoma (Figs 1, 3). Mesoscutum (L: $W=14.6: 24.5)$ finely reticulate; scutes bigger than those present on vertex; lateral pronotal area anteriorly reticulate remainder smooth; mesopleuron and metapleuron entirely smooth with distinct mesopleural and metapleural pits; scutoscutellar sulcus broad, not foveate; mesoscutellum (L: $\mathrm{W}=7.3: 17.5)$ with posterior mesoscutellar sulcus foveate; metascutellum ( $\mathrm{L}: \mathrm{W}=3.2: 9.4)$ rectangular, smooth; metanotal trough not foveate; lateral propodeal area triangular, smooth. Fore wing (L: W $=60.0: 16.5)$ and hind wing ( $\mathrm{L}: \mathrm{W}=6.7: 0.9)$ hyaline, covered with dense microtrichia; forewing with broad medial black band $0.92 \times$ width of wing; fore wing marginal cilia long, $0.31 \times$ width of wing, hind wing marginal cilia long, subequal to wing width; length of submarginalis: marginalis: stigmalis in ratio of 25.0:9.1:4.2 respectively; marginalis and stigmalis with small irregular protuberances, which is more pronounced in former than the latter (Figs 5, 6).

Metasoma. $(\mathrm{L}: \mathrm{W}=28.1: 18.0) \mathrm{T} 1$ costate medially extending $0.63 \times$ length of T1; costae medially small and increasing in size laterad; T1 with two lateral and one sublateral setae; T2 with basal foveae and short weak costae antero-medially, remainder smooth; T2 with one lateral seta; length and width of metasomal tergites T1 and T2 in ratio of 3.6:10.0, 27.4:16.9, respectively; T2 7.6x length of T1

Male: Body length $=0.93 \mathrm{~mm}(\mathrm{~m}=0.92(0.88-0.96) \mathrm{mm}, \mathrm{SD}=0.04, \mathrm{n}=20)$

Similar to female except mesoscutellum and metascutellum darker, black transverse band on posterior margin of T2 distinct.

Variation (Figs 10-13). Smaller in size $(\mathrm{m}=0.73(0.69-0.78) \mathrm{mm}, \mathrm{SD}=0.03, \mathrm{n}=9)$; reticulations on vertex, gena, mesoscutum less distinct, frons medially smooth, marginal cilia on forewing sparse (ICAR/NBAIR/P1902-P1917).

Etymology. The species name 'supattra' means having beautiful wings in Sanskrit, referring to the beautiful banded wings of the species. The name is treated as a noun in apposition.

Type material - Holotype: Female (ICAR/NBAIR/P1824), INDIA: Karnataka: Bengaluru, Jarakabande Kaval, 13.0541 N, 77.3235², 921 m, YPT, 31.VII.2013.

Paratypes: 1 female (ICAR/NBAIR/P1825), Karnataka: Bengaluru, Hesaraghatta, $13.1394^{\circ} \mathrm{N} 77.4854^{\circ} \mathrm{E}, 859 \mathrm{~m}$, YPT, 07.VII.2014; 1 female, (ICAR/NBAIR/P1826), same as P1825, 27.III.2014; 4 females (ICAR/NBAIR/P1827-P1829, P1838), Karnataka: Bengaluru, Attur, $13.0968^{\circ} \mathrm{N} 77.5641^{\circ} \mathrm{E}, 936 \mathrm{~m}, \mathrm{PFT}, 28 . \mathrm{III} .2013 ; 2$ females (ICAR/NBAIR/P1830, P1831), same as P1829, MT, 30.IV.2011; 1 female (ICAR/NBAIR/P1832), same as P1831, 07.V.2011; 1 female (ICAR/NBAIR/P1833), same as P1829, 25.I.2012; 1 female (ICAR/NBAIR/P1834), same as P1831, 17.VI.2011; 1 female (ICAR/NBAIR/P1835) same as P1831, 15.IV.2011; 1 female (ICAR/NBAIR/P1836), same as P1829, 13.IX.2011; 1 female (ICAR/NBAIR/P1837) same as P1829, YPT, 14.XI.2013; 1 female (ICAR/NBAIR/P1839), Karnataka: Bengaluru,

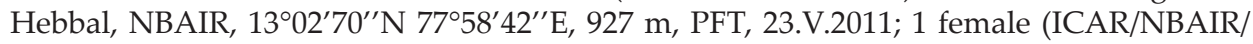
P1840), Karnataka: Gandhi Krishi Vignan Kendra (GKVK), $13.0800^{\circ} \mathrm{N} 77.5755^{\circ} \mathrm{E}, 938 \mathrm{~m}$, YPT, 29.VIII.2011; 1 female (ICAR/NBAIR/P1841), same as P1840, MT, 21.IX.2011; 1 female (ICAR/NBAIR/P1842), Karnataka: Mandya, Madduru, 12.5841N 77.0434E, 646 m, SN, 05.XI.2009; 1 female (ICAR/NBAIR/P1843), Karnataka: Mandya, Srirangapatna, $12.4232^{\circ}$ N, $76.6936^{\circ} \mathrm{E}, 676$ m, YPT, 12.V.2011; 1 female (ICAR/NBAIR/P1844), Karnataka: Kodagu, Chettalli, $12.3889^{\circ} \mathrm{N} 75.8453^{\circ} \mathrm{E}, 932 \mathrm{~m}, \mathrm{SN}, 02 . V I I .2010 ; 2$ females (ICAR/NBAIR/P1845, 

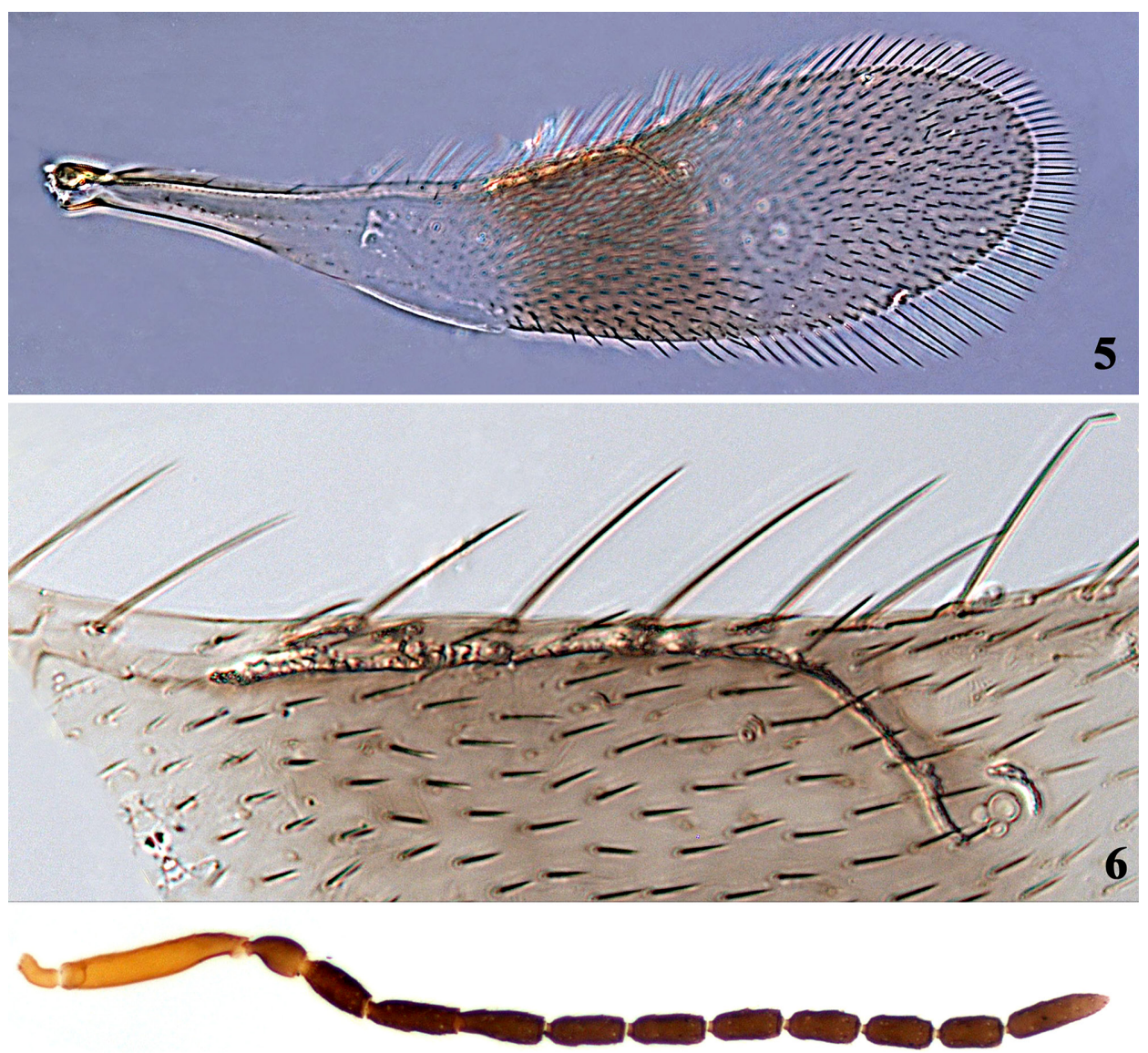

7
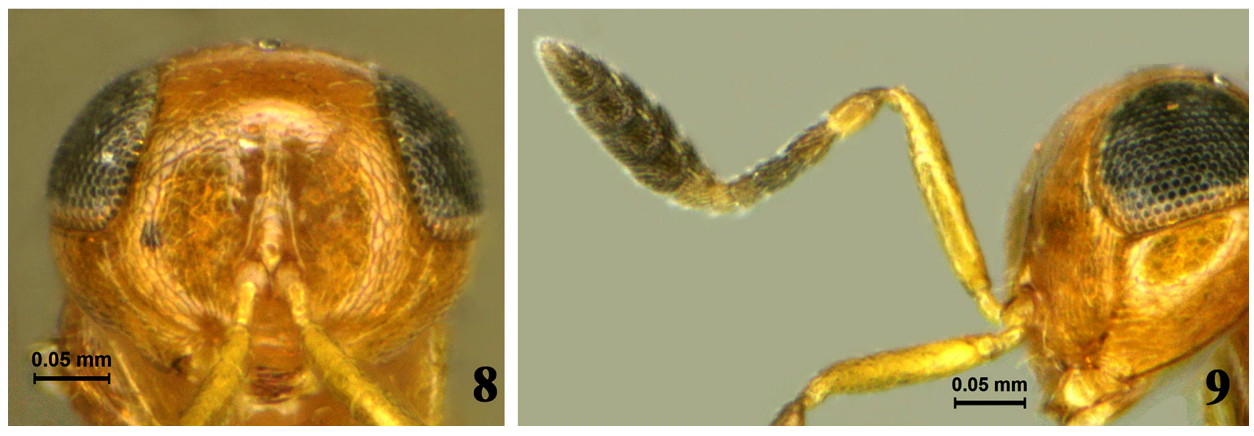

Figs 5-9. Phlebiaporus supattra sp. n. 5 = fore wing showing medial black band, $6=$ part of fore wing showing elongate marginalis and small irregular protuberances on marginalis and stigmalis, $7=$ male antenna, $8=$ frons (female), $9=$ female antenna 

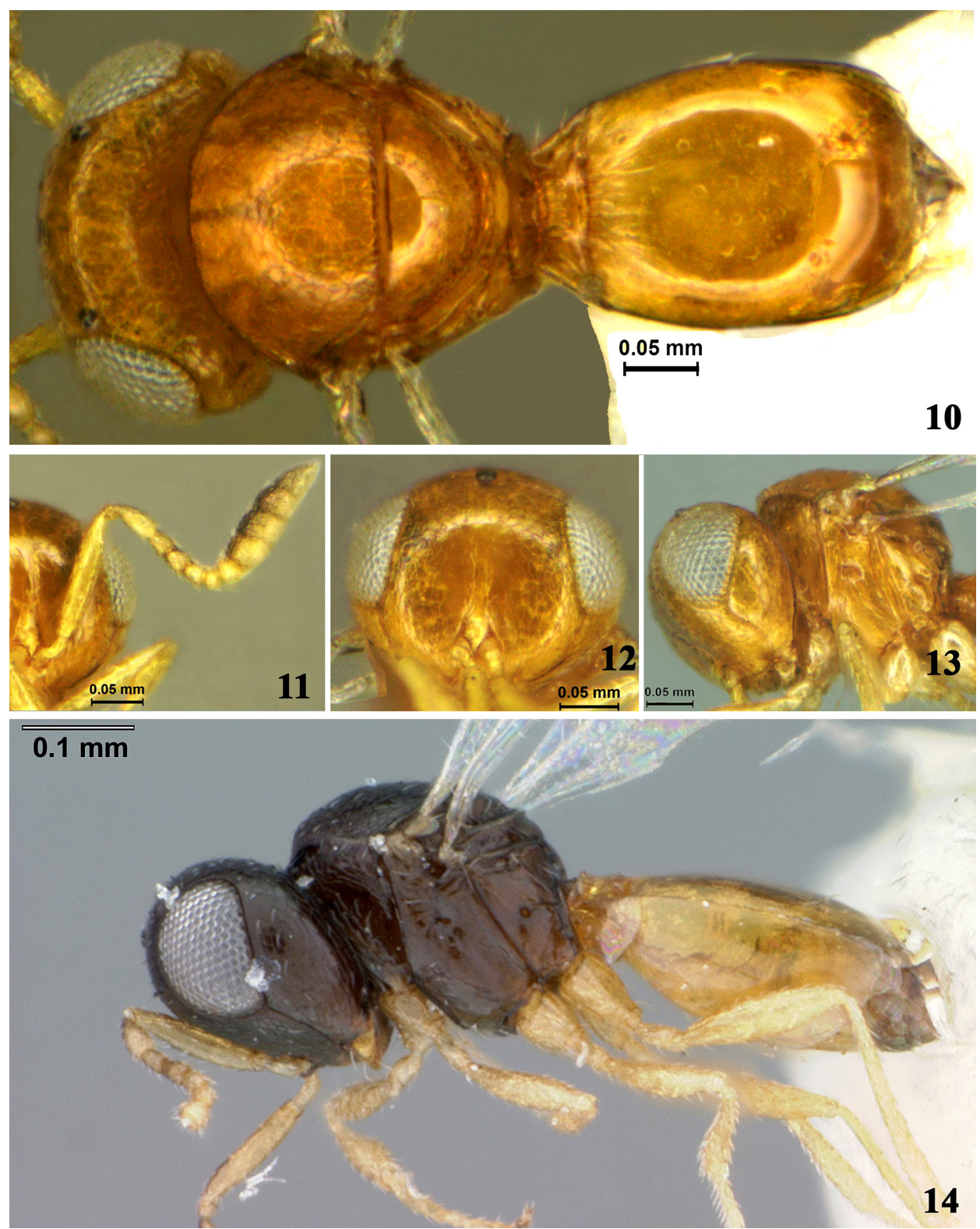

Figs 10-14. 10-13 Variant of Phlebiaporus supattra sp. n., 10 = habitus dorsal view (female), 11 = female antenna, 12 = frons showing medially smooth area, 13 = pleuron, 14 = habitus of Phlebiaporus dichrous Kozlov (paratype) (courtesy Dr. N. Johnson and E. J. Talamas, Platygastroidea Biodiversity Inventory) 
P1846), Karnataka: Bengaluru, Kengeri, $12.8999^{\circ} \mathrm{N}$ 77.4827E, 797m YPT, 17-22.XI.2012; 1 female (ICAR/NBAIR/P1847), Telangana: Hyderabad, National Academy of Agricultural Research Management (NAARM), $17.3156^{\circ} \mathrm{N} 78.4167^{\circ} \mathrm{E}, 550 \mathrm{~m}, \mathrm{SN}, 14 . \mathrm{II} .2012 ; 1$ female (ICAR/NBAIR/P1848), same as P1847, YPT; 2 females (ICAR/NBAIR/P1849, P1850), Tamil Nadu: Lower Pulney Hills, Thadiyankudisai, Horticulture Research Station (HRS), $10.2995^{\circ} \mathrm{N} 77.7117^{\circ} \mathrm{E}, 990 \mathrm{~m}, \mathrm{YPT}, 26 . X I .2016 ; 1$ female (ICAR/NBAIR/P1851), Tamil Nadu: Yercaud, Horticulture Research Station, $11.7956^{\circ} \mathrm{N} 78.2116^{\circ} \mathrm{E}, 1399$ m, YPT, 03.VIII.2016; 1 female (ICAR/NBAIR/P1852), Dharmapuri, Kelamangalam, Jakkeri, $12.6009^{\circ} \mathrm{N} 77.8489^{\circ} \mathrm{E}$, 810m YPT, 12.XI.2015; 2 females (ICAR/NBAIR/P1853, P1854), Tamil Nadu: Dharmapuri, Hosur, Uddanapalli, $12.6020^{\circ} \mathrm{N} 77.9469^{\circ} \mathrm{E}, 758 \mathrm{~m}$, MT, 28.I.2015; 1 female (ICAR/

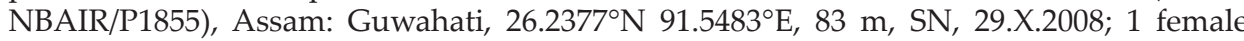
(ICAR/NBAIR/P1856), Rajasthan: Udaipur, Badagaon, $24.6274^{\circ} \mathrm{N}, 73.6823^{\circ} \mathrm{E}, 559 \mathrm{~m}, \mathrm{MT}$, 29.IX.2016; 1 female (ICAR/NBAIR/P1857), Goa: Old Goa, Ella, Central Coastal Agricultural Research Institute, $15.4933^{\circ} \mathrm{N} 73.9201^{\circ} \mathrm{E}, 15 \mathrm{~m}, \mathrm{YPT}, 14 . \mathrm{VII} .2015 ; 1$ female (ICAR/ NBAIR/P1858), Arunachal Pradesh: Pasighat, College of Horticulture and Forestry (CHF), 28.0428 ${ }^{\circ}$ 95.1928 ${ }^{\circ}$ E, 153 m, MT, 10.V.2014; 1 female (ICAR/NBAIR/P1859), Meghalaya: Ri-Bhoi, Bara Pani, Central Agricultural University (CAU), $25.7090^{\circ} \mathrm{N} 91.9514^{\circ} \mathrm{E}, 908 \mathrm{~m}$, YPT, 21.XII.2013; 9 females (ICAR/NBAIR/P1860-P1868), Himachal Pradesh: Mandi, Chandial, $31.6363^{\circ} \mathrm{N}, 76.9542^{\circ} \mathrm{E}, 774 \mathrm{~m}, \mathrm{SN}, 26 . V I .2006 ; 1$ female (ICAR/NBAIR/P1869), Punjab: Pathankot, Sali Kulian, $32.2530^{\circ} \mathrm{N} 75.6585^{\circ} \mathrm{E}, 328 \mathrm{~m}, \mathrm{SN}, 12 . \mathrm{VII} .2006 ; 1$ female (ICAR/ NBAIR/P1870), Karnataka: Chikkaballapur, Nandi Hills, $13.3702^{\circ} \mathrm{N} 77.6834^{\circ} \mathrm{E}, 1448 \mathrm{~m}$, YPT, 12.VII.2017; 1 female (ICAR/NBAIR/P1901), Karnataka: Bengaluru, Jarakabande Kaval, $13.0541^{\circ} \mathrm{N}, 77.3235^{\circ} \mathrm{E}, 921 \mathrm{~m}, \mathrm{MT}, 10 . \mathrm{VII} .2014$; 1 female (ICAR/NBAIR/P1918), same as P1839, 22.I.2010; 1 male (ICAR/NBAIR/P1871), same as P1837, 08.IV.2014; 2 males (ICAR/ NBAIR/P1872, P1873), same as P1838; 2 males (ICAR/NBAIR/P1874, P1875), same as P1837, 15.II.2014; 1 male (ICAR/NBAIR/P1876), same as P1837, 13.II.2014; 1 male (ICAR/NBAIR/ P1877), same as P1837, 14.XI.2013; 1 male (ICAR/NBAIR/P1878), same as P1837, 13.II.2014; 1 male (ICAR/NBAIR/P1879), same as P1831, 11.V.2011; 2 males (ICAR/NBAIR/ P1880, P1881), same as P1831, 07.V.2011; 1 male (ICAR/NBAIR/P1882), Karnataka: Bengaluru, Bannerghatta National Park, $12.7998^{\circ} \mathrm{N} 77.5798^{\circ} \mathrm{E}, 943$ m, SN, 10.VI.2010; 1 male (ICAR/ NBAIR/P1883), Karnataka: Bengaluru, Hesaraghatta, $13.1394^{\circ} \mathrm{N}, 77.4854^{\circ} \mathrm{E}, 859 \mathrm{~m}, \mathrm{YPT}$, 28.III.2014; 2 males (ICAR/NBAIR/P1884, P1885), Karnataka: GKVK, $13.0800^{\circ} \mathrm{N}, 77.5755^{\circ} \mathrm{E}$, 938 m, SN, 10.XII.2009; 1 male (ICAR/NBAIR/P1886), same as P1885, YPT, 22.XII.2014; 1 male (ICAR/NBAIR/P1887), Karnataka: Bengaluru, Hebbal, Indian Veterinary Research Institute (IVRI), $13.0247^{\circ} \mathrm{N} 77.5834^{\circ} \mathrm{E}, 932 \mathrm{~m}$, YPT, 05.V.2015; 1 male (ICAR/NBAIR/P1888), same as holotype, 18.VIII.2014; 5 males (ICAR/NBAIR/P1889-1893), same as 1901; 2 males (ICAR/NBAIR/P1894, P1895), same as P1837, 30.V.2011; 1 male (ICAR/NBAIR/P1896), same as P1842, MT, 07.VI.2012; 1 male (ICAR/NBAIR/P1897), same as P1868; 2 males (ICAR/NBAIR/P1898, P1899), Madhya Pradesh: Bhopal, Central Institute of Agricultural Engineering (CIAE), 23.3086 ${ }^{\circ} \mathrm{N} 77.4016^{\circ} \mathrm{E}, 499 \mathrm{~m}, \mathrm{SN}, 16 . X \mathrm{XI} .2011 ; 2$ males (ICAR/NBAIR/ P1900) same as P1899, YPT, 15.XII.2011;1 female (ICAR/NBAIR/P1902), Karnataka: Ghatti Subramanya, $1^{3} .4081^{\circ} \mathrm{N} 77.5279^{\circ} \mathrm{E}, 883 \mathrm{~m}$, YPT, 31.I.2014; 1 female (ICAR/NBAIR/P1903), same P1845, 26.X.2012; 1 female (ICAR/NBAIR/P1904), Karnataka: Chikkaballapur, Nandi Hills, $13.3702^{\circ} \mathrm{N}, 77.6834^{\circ} \mathrm{E}, 1448 \mathrm{~m}$, YPT, 05.VII.2013; 1 female (ICAR/NBAIR/P1905), same as P1904, PFT, 8.IX.2010; 2 females (ICAR/NBAIR/P1906, P1907), same as P1904, SN, 10.IX.2010; 1 female ICAR/NBAIR/P1908), same as P1842, MT, 07.VIII.2012; 1 female (ICAR/NBAIR/P1909), same as P1852, 17.IV.2015; 1 female (ICAR/NBAIR/P1910), same as P1900; 1 female (ICAR/NBAIR/P1911), same as P1900, High Security Animal Disease Laboratory (HSADL), 16.XII.2011; 1 male (ICAR/NBAIR/P1912), same as P1904, 05.X.2010; 1 
male (ICAR/NBAIR/P1913), same as P1907, 08.IX.2010; 1 male (ICAR/NBAIR/P1914), same as P1842, MT, 07.VIII.2012; 1 male (ICAR/NBAIR/P1915), Karnataka: Bengaluru, Hebbal, NBAIR, 13.0270N 77.5842 ${ }^{\circ}$, 927 m, MT, 18.IV.2011; 1 male (ICAR/NBAIR/P1916), same as P1900; 1 male (ICAR/NBAIR/P1917), Uttar Pradesh; Lucknow, Indian Institute of Sugarcane Research (IISR), $26.8051^{\circ} \mathrm{N} 80.2336^{\circ} \mathrm{E}, 124 \mathrm{~m}, \mathrm{SN}, 13 . \mathrm{II} .2011$.

Acknowledgements - The authors are grateful to the Director, NBAIR, Bengaluru for providing facilities and encouragement. We thank B. L. Lakshmi, V. Shashikala, B. Vinod and Roopa for their support both in the field and laboratory. Thanks are also due to the Platygastroidea Planetary Biodiversity Inventory, under National Science Foundation grant No. DEB-0614764, for literature support. Special thanks to Dr. E. J. Talamas for taxonomic discussion. We are grateful to Dr. N. F. Johnson and Dr. E. J. Talamas for the images of the paratype of $P$. dichrous hosted in Specimage. Sincere thanks to Dr. Sunil Joshi and Dr. Jagadish Patil for images of the wings. We are also grateful to Dr. Swaminathan Iyer for his kind help.

\section{REFERENCES}

Johnson, N. F. (1992): Catalog of world Proctotrupoidea excluding Platygastridae. - Memoirs of American Entomological Institute 51:1-825. https://doi.org/10.5281/zenodo.23657

Johnson, N. F. (2017): Platygastroidea. - Available from: http://osuc.biosci.ohio-state.edu/ hymDB/eol_scelionidae.home (Accessed on 1.3.2017)

Kozlov, M. A. (1967): [New representatives of Proctotrupoidea (Hymenoptera) from Kazakhstan and Middle Asia.] - Zoologicheskii Zhurnal 46: 715-720. https://doi. org/10.5281/zenodo. 23963

Kozlov, M. A. \& Kononova, S. V. (1983): [Telenominae of the fauna of the USSR.] - Nauka, Leningrad. $336 \mathrm{pp}$.

MAsner, L. (1976): Revisionary notes and keys to world genera of Scelionidae (Hymenoptera: Proctotrupoidea). - Memoirs of Entomological Society of Canada 108(S97): 1-87. https://doi.org/10.4039/entm10897fv

MAsner, L. (1980): Key to genera of Scelionidae of the Holarctic region, with descriptions of new genera and species (Hymenoptera: Proctotrupoidea). - Memoirs of Entomological Society of Canada 112(S113): 1-54. https://doi.org/10.4039/entm112113fv

Miкó, I., Masner, L. \& Deans, A. R. (2010): World revision of Xenomerus Walker (Hymenoptera: Platygastroidea, Platygastridae). - Zootaxa 2708: 1-73.

Mikó, I., Vilhelmsen, L., Johnson, N. F., Masner, L. \& Pénzes, Z. (2007): Skeleto-musculature of Scelionidae (Hymenoptera: Platygastroidea) head and mesosoma. - Zootaxa 1571: 1-78.

Mikó, I., Trietsch, C., Sandall, E. L., Yoder, M. J., Hines, H. \& Deans, A. R. (2016): Malagasy Conostigmus (Hymenoptera: Ceraphronoidea) and the secret of scutes. PeerJ 4:e2682. https://doi.org/10.7717/peerj.2682

Received July 27, 2017, accepted June 11, 2017, published August 1, 2018 